\title{
An Unconditionally Stable Staggered Algorithm for Transient Finite Element Analysis of Coupled Thermoelastic Problems
}

C. Farhat and K.C. Park

University of Colorado

Boulder, Colorado

and

Y. Dubois-Pelerin

Ecole Polytechnique Federale de Lausanne

Lausanne, Switzerland

November 1991

Prepared for

Lewis Research Center

Under Grants NAGW - 1388 and NAG3-934

\section{N/SA \\ National Aeronautics and \\ Space Administration}

(HASA-CR-169066) AN WIVONDITIONALLY STADLE

N92-13457

STAGGFRFD ALGORITHM FDR TRANSIENT FINITE

ELEMENT ANALYSIS OF CNUPLED THERMNELASTIC

PRDLLFS Final Report, Jul. 1989 (Colorado

Univ.) 260 


\title{
AN UNCONDITIONALLY STABLE STAGGERED ALGORITHM FOR TRANSIENT FINITE ELEMENT ANALYSIS OF COUPLED THERMOELASTIC PROBLEMS
}

\author{
C. Farhat, K.C. Park \\ University of Colorado \\ Department of Aerospace Engineering Sciences \\ Boulder, CO 80309 \\ and \\ Y. Dubois-Pelerin \\ Ecole Polytechnique Federale de Lausanne \\ Department of Civil Engineering \\ Lausanne, Switzerland
}

\begin{abstract}
An unconditionally stable second order accurate implicit-implicit staggered procedure for the finite element solution of fully coupled thermoelasticity transient problems is proposed. The procedure is stabilized with a semialgebraic augmentation technique. A comparative cost analysis reveals the superiority of the proposed computational strategy to other conventional staggered procedures. Numerical examples of one and two-dimensional thermomechanical coupled problems demonstrate the accuracy of the proposed numerical solution algorithm.
\end{abstract}

\section{INTRODUCTION}

Transient response prediction of thermally loaded structures is of considerable importance in many aerospace engineering problems, and it has been the subject of intense research. Finite element formulations of the classical heat conduction problem without mechanical coupling have been presented by Wilson and Nickell [1]. Ritz type methods for the solution of linear dynamic problems in coupled thermoelasticity were given by Nickell and Sackman [2]. Oden [3] has formulated finite element models for the analysis of a class of nonlinear problems in dynamic coupled thermoelasticity, and Oden and Armstrong [4] have developed explicit 
quadratic numerical schemes for the integration of nonlinear unpartitioned systems of difference equations arising from the analysis of dynamic coupled thermoviscoelastic problems. Recently, Ting and Chen [5] have introduced a unified numerical approach for the analysis of thermal stress waves. They have derived their algorithm from the concept of heat displacement and a variational formulation in Lagrangian form. They have proposed to integrate the resulting semidiscrete equations with conditionally stable explicit schemes. Liu and Zhang [6] have described an implicit-explicit procedure for the prediction of thermal stress waves in coupled thermoelasticity problems. They have adopted the explicit rational Runge-Kutta method $[7,8]$ for approximately solving the heat conduction equation and have claimed that their solution procedure is unconditionally stable. However, their computational strategy requires the manipulation of a full matrix. In a sequel note, Liu and Chang [9] have slightly modified the original procedure of Liu and Zhang to involve a banded rather than full matrix, and have numerically verified the unconditional stability on one dimensional problems.

However, several practical issues must be resolved before unconditionally stable explicit rational Runge-Kutta schemes can become suitable for the analysis of real thermomechanical coupled problems. First, when unconditional stability is achieved for explicit time integration algorithms, typically consistency becomes conditional (see for example Hughes and Belytschko [10]). Second, most rational Runge-Kutta algorithms involve some divide operations by the difference between intermediate solution quantities, which can significantly damage accuracy. Finally, these algorithms do not appear to accomodate staggered solution procedures for thermal/structure interaction problems, as they are not implemented in many existing production-level thermal computer programs.

The semi-discrete equations governing soil-pore fluid interaction dynamic problems are similar to those governing thermoelastic coupled transient problems. In this sense, the work of Park [11], and very recently that of Zienkiewicz, Paul and Chan [12] can be extended to the response analysis of thermally loaded structures.

In the present work, we present an unconditionally stable and robust implicitimplicit partitioned procedure for the solution of transient thermoelastic coupled problems. In Section II, we briefly review the basic equations for the linearized coupled thermoelasticity theory. A conventional implicit-implicit staggered solution procedure is summarized in Section III. The thermal coupling term in the structural dynamics equation is treated as an applied force. However, while being very simple to implement, the resulting time integration algorithm suffers from 
conditional stability. In Section IV, we introduce an augmented implicit-implicit staggered solution procedure for the partitioned problem. We establish the unconditional stability and second order accuracy of the resulting numerical algorithm in Section V. In Section VI, we discuss the computer implementation aspects of the proposed computational strategy; we conduct a comparative cost analysis which demonstrates the superiority of the proposed solution procedure to other conventional staggered schemes. Finally in Section VII, we apply our partitioned algorithm to the solution of the one-dimensional Second Danilovskaya [13] and two-dimensional Youngdahl-Stemberg [14] problems. For both problems, the results generated by the proposed stabilized procedure are shown to be in excellent agreement with the analytical "exact" solutions.

\section{FINITE ELEMENT FORMULATION}

Let $B$ denote the body of the structure to be analyzed, and $\partial B=\partial B_{u} \cup \partial B_{s} \cup$ $\partial B_{\theta} \cup \partial B_{q}$ the surface enclosing it. The basic equations for the linearized isotropic coupled thermoelasticity theory are:

$$
\begin{aligned}
\rho \ddot{\mathbf{u}} & =\operatorname{div} \boldsymbol{\sigma}+\mathbf{b} \text { in } B \\
c \dot{\boldsymbol{\theta}} & =-\operatorname{div}(-k \nabla \boldsymbol{\theta})-\alpha(3 \lambda+2 \mu) \theta_{0} \operatorname{tr}(\dot{\boldsymbol{\epsilon}})+\mathbf{r} \text { in } B \\
\boldsymbol{\sigma} & =2 \mu \boldsymbol{\epsilon}+\lambda(\operatorname{tr} \boldsymbol{\epsilon}) \mathbf{I}-\alpha(3 \lambda+2 \mu)\left(\boldsymbol{\theta}-\theta_{0}\right) \mathbf{I} \\
\boldsymbol{\epsilon} & =\frac{1}{2}\left(\nabla \mathbf{u}+\nabla \mathbf{u}^{T}\right)
\end{aligned}
$$

and

$$
\begin{aligned}
\mathbf{u}=\hat{\mathbf{u}} & \text { on } \partial B_{u} \\
\boldsymbol{\sigma} \mathbf{n}=\hat{\mathbf{s}} & \text { on } \partial B_{s} \\
\boldsymbol{\theta}=\hat{\theta} & \text { on } \partial B_{\theta} \\
-k \nabla \boldsymbol{\theta}=\hat{q} & \text { on } \partial B_{q}
\end{aligned}
$$

where $\mathbf{u}, \boldsymbol{\epsilon}, \boldsymbol{\sigma}, \theta, \theta_{0}, \mathbf{b}$, and $r$ are the displacement, strain, stress, temperature, reference temperature chosen such that $\left(\theta-\theta_{0}\right) / \theta_{0}<<1$, body force, and heat supply fields, respectively, while $\mu, \lambda, c, \alpha, \rho, k$ and $\mathbf{n}$ are the Lame' moduli, the shear modulus, the specific heat, the coefficient of thermal expansion, the mass per unit volume, the thermal diffusivity, and the normal to the surface at a given 
point, respectively. $\mathbf{I}$ is the identity tensor. The dot and $T$ superscripts denote a time derivative and a transpose operation, and $t r$ denotes the trace of a given tensor.

If now we express the dependent variables $\mathbf{u}$ and $\theta$ by suitable shape functions as:

$$
\mathbf{u}=\mathbf{N} \bar{u} \text { and } \theta=\hat{N} \bar{\theta}
$$

then a standard Galerkin procedure transforms (1) in the following algebraic coupled system of differential equations:

$$
\begin{aligned}
\mathbf{M} \ddot{\mathbf{u}}+\mathbf{D} \dot{\mathbf{u}}+\mathbf{K u}-\mathbf{C} \boldsymbol{\theta} & =\mathbf{f} \\
\mathbf{Q} \dot{\theta}+\mathbf{H} \boldsymbol{\theta}+\theta_{0} \mathbf{C}^{T} \dot{\mathbf{u}} & =\mathbf{r}
\end{aligned}
$$

where $\mathbf{M}, \mathbf{D}$ and $\mathbf{K}$ are the usual mass, damping and stiffness matrices, $\mathbf{f}$ is the prescribed structural loading vector, and $\mathbf{Q}, \mathbf{H}$, and $\mathbf{r}$ are respectively the capacity and conductivity matrices and the nodal source vector. If $L$ denotes the differential operator corresponding to strain, the coupling matrix is expressed as $\mathbf{C}=\int_{B}(\mathbf{L N})^{T}[1,1,1,0,0,0] \hat{N} d B$

\section{CONVENTIONAL IMPLICIT-IMPLICIT PROCEDURE}

In many applications, the coupling term $\mathbf{C}^{T} \dot{\mathbf{u}}$ that appears in the heat equation and which is induced by the effect of the strain rate is negligible. Therefore, one expects the second of equations (2) to remain parabolic and the temperature response to remain close to the uncoupled solution. Consequently, the dependent variable $\theta$ is easier to predict than the displacement $\mathbf{u}$, so that the most natural way of solving (2) would be:

$$
\begin{aligned}
\mathrm{M} \ddot{\mathbf{u}}^{n+1}+\mathrm{D} \dot{\mathbf{u}}^{n+1}+\mathbf{K} \mathbf{u}^{n+1} & =\mathbf{f}^{n+1}+\mathbf{C} \theta^{n+1} P \\
\mathbf{Q} \dot{\theta}^{n+1}+\mathbf{H} \theta^{n+1} & =\mathbf{r}^{n+1}-\theta_{0} \mathbf{C}^{T} \dot{\mathbf{u}}^{n+1}
\end{aligned}
$$

where $\theta^{n+1} P$ is the predicted temperature. Unfortunately, the above numerical procedure is only conditionally stable, even when each field is integrated with an unconditionally stable algorithm. Proofs of this result are given by DuboisPelerin [15] for various consistent predictors. Next, we introduce an augmentation technique that stabilizes the staggered solution of (2). 


\section{AN AUGMENTED IMPLICIT-IMPLICIT PARTITIONED PRO- CEDURE}

Park, Felippa and DeRuntz [16] have introduced a differential augmentation concept that was successfully used in the stabilization of staggered solution procedures for fluid-structure interaction problems. Basically, one of the coupled equations is injected into the other in order to "soften" the system, either by reducing the large eigenvalues of the uncoupled stiff equation, or by introducing some damping into it. Here, we adopt a different strategy. We perform a semialgebraic augmentation - that is, we augment one of the two coupled equations while integrating both fields.

First, the structural equation is integrated with the trapezoidal rule:

$$
\begin{aligned}
\dot{\mathbf{u}}^{n+1} & =\dot{\mathbf{u}}^{n}+\frac{\Delta t}{2}\left(\ddot{\mathbf{u}}^{n+1}+\ddot{\mathbf{u}}^{n}\right) \\
& =\dot{\mathbf{u}}^{n}+\frac{\Delta t}{2}\left[\ddot{\mathbf{u}}^{n}+\mathbf{M}^{-1}\left(\mathbf{f}^{n+1}-\mathrm{D} \dot{\mathbf{u}}^{n+1}-\mathbf{K} \mathbf{u}^{n+1}+\mathbf{C} \theta^{n+1}\right)\right] \\
\mathbf{u}^{n+1} & =\mathbf{u}^{n}+\frac{\Delta t}{2}\left(\dot{\mathbf{u}}^{n+1}+\dot{\mathbf{u}}^{n}\right) \\
& =\mathbf{u}^{n}+\Delta t \dot{\mathbf{u}}^{n}+\frac{\Delta t^{2}}{4}\left[\ddot{\mathbf{u}}^{n}+\mathbf{M}^{-1}\left(\mathbf{f}^{n+1}-\mathbf{D} \dot{\mathbf{u}}^{n+1}-\mathbf{K} \mathbf{u}^{n+1}+\mathbf{C} \theta^{n+1}\right)\right]
\end{aligned}
$$

and the velocity vector is extracted as:

$$
\left(\mathbf{I}+\frac{\Delta t}{2} \mathbf{M}^{-1} \mathbf{D}\right) \dot{\mathbf{u}}^{n+1}=\dot{\mathbf{u}}^{n}+\frac{\Delta t}{2}\left[\ddot{\mathbf{u}}^{n}+\mathbf{M}^{-1}\left(\mathbf{f}^{n+1}-\mathbf{K} \mathbf{u}^{n+1}+\mathbf{C} \theta^{n+1}\right)\right]
$$

Next, the heat equation is also integrated with the trapezoidal rule:

$$
\begin{aligned}
\theta^{n+1} & =\theta^{n}+\frac{\Delta t}{2}\left(\dot{\theta}^{n+1}+\dot{\theta}^{n}\right) \\
& =\theta^{n}+\frac{\Delta t}{2}\left[\dot{\theta}^{n}+\mathbf{Q}^{-1}\left(\mathbf{r}^{n+1}-\mathbf{H} \theta^{n+1}-\theta_{0} \mathbf{C}^{T} \dot{\mathbf{u}}^{n+1}\right)\right]
\end{aligned}
$$

Finally, the system is augmented by recasting (5) in (6) to obtain: 


$$
\begin{aligned}
\theta^{n+1} & =\theta^{n}+\frac{\Delta t}{2}\left\{\dot{\boldsymbol{\theta}}^{n}+\mathbf{Q}^{-1}\left[\mathbf{r}^{n+1}-\mathbf{H} \theta^{n+1}-\theta_{0} \mathbf{C}^{T}\left(\mathbf{I}+\frac{\Delta t}{2} \mathbf{M}^{-1} \mathbf{D}\right)^{-1}\right.\right. \\
& \left.\left.\left(\dot{\mathbf{u}}^{n}+\frac{\Delta t}{2}\left(\ddot{\mathbf{u}}^{n}+\mathbf{M}^{-1}\left(\mathbf{f}^{n+1}-\mathbf{K} \mathbf{u}^{n+1}+\mathbf{C} \boldsymbol{\theta}^{n+1}\right)\right)\right)\right]\right\}
\end{aligned}
$$

Substituting (5) into the second of equations (4) and re-arranging (7) leads to:

$$
\begin{aligned}
&\left(\mathbf{I}+\frac{\Delta t^{2}}{4} \mathbf{B}(\Delta t) \mathbf{M}^{-1} \mathbf{K}\right) \mathbf{u}^{n+1}-\frac{\Delta t^{2}}{4} \mathbf{B}(\Delta t) \mathbf{M}^{-1} \mathbf{C} \theta^{n+1}=\mathbf{F}^{n+1} \\
&\left(-\frac{\Delta t^{2}}{4} \theta_{0} \mathrm{AK}\right) \mathbf{u}^{n+1}+\left(\mathbf{I}+\frac{\Delta t}{2} \mathbf{Q}^{-1} \mathbf{H}+\frac{\Delta t^{2}}{4} \theta_{0} \mathbf{A C}\right) \theta^{n+1}=\mathbf{R}^{n+1}
\end{aligned}
$$

where

$$
\begin{aligned}
\mathbf{A} & =\mathbf{Q}^{-1} \mathbf{C}^{T} \mathbf{M}^{-1} \\
\mathbf{B}(\Delta t) & =\left(\mathbf{I}+\frac{\Delta t}{2} \mathbf{M}^{-1} \mathbf{D}\right)^{-1} \\
\mathbf{F}^{n+1} & =\mathbf{u}^{n}+\frac{\Delta t}{2}(\mathbf{I}+\mathbf{B}(\Delta t)) \dot{\mathbf{u}}^{n}+\frac{\Delta t^{2}}{4}\left(\mathbf{B}(\Delta t) \ddot{\mathbf{u}}^{n}+\mathbf{M}^{-1} \mathbf{B}(\Delta t) \mathbf{f}^{n+1}\right) \\
\mathbf{R}^{n+1} & =\theta^{n}+\frac{\Delta t}{2}\left[\dot{\theta}^{n}+\mathbf{Q}^{-1}\left(\mathbf{r}^{n+1}-\theta_{0} \mathbf{C}^{T} \mathbf{B}(\Delta t) \dot{\mathbf{u}}^{n}\right)\right] \\
& -\frac{\Delta t^{2}}{4}\left[\theta_{0} \mathbf{Q}^{-1} \mathbf{C}^{T}\left(\mathbf{B}(\Delta t) \ddot{\mathbf{u}}^{n}+\mathbf{B}(\Delta t) \mathbf{M}^{-1} \mathbf{f}^{n+1}\right)\right]
\end{aligned}
$$

Now, a displacement predicted staggered procedure for the solution of $(S)$ is:

1. Predict the displacement field:

$$
\mathbf{u}^{n+1} P=\mathbf{u}^{n}
$$

2. Solve for the temperature field:

$$
\left(\mathbf{I}+\frac{\Delta t}{2} \mathbf{Q}^{-1} \mathbf{H}+\frac{\Delta t^{2}}{4} \theta_{0} \mathrm{AC}\right) \theta^{n+1}=\mathrm{R}^{n+1}+\frac{\Delta t^{2}}{4} \theta_{0} \mathrm{AK} \mathrm{\mathbf {u } ^ { n + 1 } P}
$$


3. Correct the displacement field:

$$
\left(\mathbf{I}+\frac{\Delta t^{2}}{4} \mathbf{B}(\Delta t) \mathbf{M}^{-1} \mathbf{K}\right) \mathbf{u}^{n+1}=\mathbf{F}^{n+1}+\frac{\Delta t^{2}}{4} \mathbf{B}(\Delta t) \mathbf{M}^{-1} \mathbf{C} \theta^{n+1}
$$

4. Compute velocity, acceleration and flux fields:

$$
\begin{aligned}
\dot{\mathbf{u}}^{n+1} & =\mathrm{B}(\Delta t)\left\{\dot{\mathbf{u}}^{n}+\frac{\Delta t}{2}\left[\ddot{\mathbf{u}}^{n}+\mathrm{M}^{-1}\left(\mathbf{f}^{n+1}-\mathbf{K} \mathbf{u}^{n+1}+\mathbf{C} \theta^{n+1}\right)\right]\right\} \\
\ddot{\mathbf{u}}^{n+1} & =\mathbf{M}^{-1}\left(\mathbf{f}^{n+1}+\mathbf{C} \theta^{n+1}-\mathbf{D} \dot{\mathbf{u}}^{n+1}-\mathbf{K} \mathbf{u}^{n+1}\right) \\
\dot{\theta}^{n+1} & =\mathbf{Q}^{-1}\left(\mathbf{r}^{n+1}-\theta_{0} \mathbf{C}^{T} \dot{\mathbf{u}}^{n+1}-\mathbf{H} \theta^{n+1}\right)
\end{aligned}
$$

\section{Remarks:}

1. the predictor $\mathbf{u}^{n+1} P$ is simply the previous step solution. It has been found (see Park [17]) that this is the most stable predictor when used in conjunction with the trapezoidal rule, while still maintaining a second-order accuracy.

2. the injection of (5) into (6) is not arbitrary. It will be shown in Section VI that this is more economical than injecting (6) into (5).

3. equations (13) define the computational path of the staggered procedure.

\section{STABILITY AND ACCURACY ANALYSES}

In this section, we establish that equations (10)-(13) result in a unconditionally stable second order accurate transient algorithm for the time integration of the coupled system (2). To avoid lengthy expressions, we consider the undamped $(\mathbf{D}=0)$ and unforced $(\mathbf{f}=\mathbf{r}=0)$ case. Note however that even when $\mathbf{D}=0$, the quantity $\mathbf{C} \theta$ still transmits a rate dependent damping effect to the structural equation.

Stability. The stability of the proposed staggered procedure can be examined by seeking a nontrivial solution in the form:

$$
\left[\begin{array}{c}
\mathbf{u}^{n+1} \\
\dot{\mathbf{u}}^{n+1} \\
\ddot{\mathbf{u}}^{n+1} \\
\theta^{n+1} \\
\dot{\theta}^{n+1}
\end{array}\right]=\frac{1+z}{1-z}\left[\begin{array}{c}
\mathbf{u}^{n} \\
\dot{\mathbf{u}}^{n} \\
\ddot{\mathbf{u}}^{n} \\
\theta^{n} \\
\dot{\theta}^{n}
\end{array}\right]
$$


and determining under what condition the real part of $z$ is positive. Substitution of (10) into (11) and (14) into (11)-(13) yields, after some algebraic manipulations:

$$
\left[\begin{array}{cc}
z^{2} \mathbf{I}+\frac{\Delta t^{2}}{4} \mathbf{M}^{-1} \mathrm{~K} & -\frac{\Delta t^{2}}{4} \mathbf{M}^{-1} \mathbf{C} \\
-\left(1-z^{2}\right) \frac{\Delta t^{2}}{4} \theta_{0} \mathrm{AK} & z^{2} \mathrm{I}+z \frac{\Delta t}{2} \mathbf{Q}^{-1} \mathbf{H}+\frac{\Delta t^{2}}{4} \theta_{0} \mathbf{A C}
\end{array}\right]\left[\begin{array}{l}
\mathbf{u}^{n} \\
\theta^{n}
\end{array}\right]=\left[\begin{array}{l}
0 \\
0
\end{array}\right]
$$

Therefore, the characteristic equation associated with (15) is:

$$
\left|\mathbf{M} z^{3}+\mathrm{VM} \frac{\Delta t}{2} z^{2}+\left(\mathrm{K}+\theta_{0} \mathbf{C Q}^{-1} \mathbf{C}^{T}+\frac{\Delta t^{2}}{4} \theta_{0} \mathbf{C Q}^{-1} \mathbf{C}^{T} \mathbf{M}^{-1} \mathrm{~K}\right) \frac{\Delta t^{2}}{4} z+\mathrm{VK} \frac{\Delta t^{3}}{8}\right|=0
$$

where

$$
\begin{aligned}
\mathbf{V} & =\mathbf{C U C} \\
\mathbf{U} & =\mathbf{Q}^{-1} \mathbf{H}\left(\mathbf{C}^{T} \mathbf{C}\right)^{-1}
\end{aligned}
$$

and || denotes the matrix determinant. If the matrices $\mathbf{M}, \mathbf{K}, \mathbf{Q}$ and $\mathbf{H}$ are positive definite, and the coupling matrix $\mathbf{C}$ has full column rank, then $\mathbf{U}, \mathbf{V}$ and each matrix coefficient of the determinant expression (16) is positive definite. If $\mathbf{C}$ is column rank deficient, $\mathbf{U}$ and $\mathbf{V}$ are positive semi-definite. In any case, all coefficients of the stability polynomial are non-negative. Consequently, the first part of the Routh-Hurwitz criterion [18] for unconditional stability is satisfied. In order to check the second component of this criterion, we consider a 2-d.o.f. model problem for (2). The corresponding scalar form of (16) is:

$$
a_{3} z^{3}+a_{2} z^{2}+a_{1} z+a_{0}=0
$$

where

$$
a_{3}=1, \quad a_{2}=\frac{\Delta t h}{2 q}, \quad a_{1}=\frac{\Delta t^{2}}{4}\left[\omega^{2}+\frac{\theta_{0} c^{2}}{q m}\left(1+\frac{\Delta t^{2}}{4} \omega^{2}\right)\right], \quad a_{0}=\frac{\Delta t^{3} h}{8 q} \omega^{2}
$$

Since $\Delta t, h, q, w^{2}, \theta_{0}, c^{2}$, and $m>0$, then all the coefficients of the polynomial (17) in $z$ are positive. Morevoer, the quantity

$$
a_{1} a_{2}-a_{0} a_{3}=\frac{\theta_{0} h c^{2} \Delta t^{3}}{8 m q^{2}}\left(1+\frac{\Delta t^{2}}{4} w^{2}\right)
$$


is also positive, which demonstrates that the staggered solution procedure is unconditionally stable for the 2-d.o.f. model problem.

For the general multi-dimensional case, it turns out that the limiting case $\mathbf{K}=\mathbf{0}$ which states that the structural system will grow quadratically in time, provides a sufficient test. For this case, (16) reduces to:

$$
\left|\mathbf{M} z^{2}+\frac{\Delta t}{2} \mathrm{VM} z+\frac{\Delta t^{2}}{4} \theta_{0} \mathrm{CQ}^{-1} \mathbf{C}^{T}\right|=0
$$

Since $\mathbf{M}$ is positive definite and $\mathrm{VM}$ and $\mathbf{C Q}^{-1} \mathbf{C}^{T}$ are at least positive semidefinite, the procedure is unconditionally stable for the limiting case $K=0$, as discussed in Bellman [19]. This argument has been extensively utilized in [12] during the analysis of several partitioned procedures. Therefore, we conclude that the procedure given by (10)-(13) is unconditionally stable.

\section{Remarks:}

1. the characteristic equation (16) reveals that the proposed procedure (10)(13) is algorithmically identical to the one obtained by first differentiating the second of equations (2):

$$
\mathrm{Q} \ddot{\theta}+\mathbf{H} \ddot{\theta}+\theta_{0} \mathbf{C}^{T} \ddot{\mathbf{u}}=\dot{\mathbf{r}}
$$

then substituting $\ddot{\mathbf{u}}$ from the first of equations (2) into the above equation:

$$
\mathbf{Q} \ddot{\theta}+\mathbf{H} \dot{\theta}+\theta_{0} \mathrm{C}^{T} \mathbf{M}^{-1} \mathbf{C} \theta==\dot{\mathbf{r}}-\theta_{0} \mathbf{C}^{T} \mathbf{M}^{-1}(\mathbf{f}-\mathbf{K u})
$$

However, differentiating the nodal source vector may be not practical, for example, if $\mathbf{r}$ is a discontinuous function of time. In our present derivation (11)-(13) we avoid this problem.

2. the first-order thermal equation is algorithmically modified to behave as a damped second-order system. It should be emphasized that the described stabilization technique has not introduced any artificial damping. The only augmentation that is used is part of the governing equation of motion itself. 
Accuracy. After differentiation, the third of equations (13) in the unforced case reads:

$$
\ddot{\theta}^{n+1}=-\theta_{0} \mathbf{Q}^{-1} \mathbf{C}^{T} \ddot{\mathbf{u}}^{n+1}-\mathbf{Q}^{-1} \mathbf{H} \dot{\theta}^{n+1}
$$

Expanding the various terms in (8) around the time $n \Delta t$ and injecting (13) and (18) when needed leads to:

$$
\begin{aligned}
\mathbf{M} \ddot{\mathbf{u}}^{n}+\mathbf{K} \mathbf{u}^{n} & =\mathbf{C} \theta^{n}+O\left(\Delta t^{2}\right) \\
\mathbf{Q} \dot{\theta}^{n}+\mathbf{H} \theta^{n} & =-\theta_{0} \mathbf{C}^{T} \dot{\mathbf{u}}^{n}+O\left(\Delta t^{2}\right)
\end{aligned}
$$

Comparing (2) and (19) demonstrates that the staggered procedure is second order accurate. The same result can be proved for the damped $(\mathbf{D} \neq 0)$ and forced $(\mathbf{f} \neq 0, \mathbf{r} \neq 0)$ case.

\section{COMPUTATIONAL ASPECTS}

In the remainder of this paper, we consider the case where the structure is undamped $(\mathbf{D}=0)$ and the mass and capacity matrices are lumped $(\mathbf{M}, \mathbf{Q}$ are diagonal). The unconditionally stable staggered procedure (10)-(13) can be implemented as:

1. Form:

$$
\mathbf{R}^{n+1}=\frac{\Delta t}{2} \mathbf{r}^{n+1}+\mathbf{Q}\left[\theta^{n}+\frac{\Delta t}{2}\left(\dot{\theta}^{n}-\theta_{0} \mathbf{C}^{T} \dot{\mathbf{u}}^{n}\right)\right]-\frac{\Delta t^{2}}{4} \theta_{0} \mathbf{C}^{T}\left[\ddot{\mathbf{u}}^{n}+\mathrm{M}^{-1}\left(\mathbf{f}^{n+1}-\mathrm{Ku}^{n+1} P\right)\right]
$$

2. Solve:

$$
\left(\mathbf{Q}+\frac{\Delta t}{2} \mathbf{H}+\frac{\Delta t^{2}}{4} \theta_{0} \mathrm{C}^{T} \mathbf{M}^{-1} \mathrm{C}\right) \theta^{n+1}=\mathrm{R}^{n+1} *
$$

3. Form:

$$
\mathbf{F}^{n+\mathbf{l}^{*}}=\mathbf{M}\left[\mathbf{u}^{n}+\Delta t\left(\dot{\mathbf{u}}^{n}+\frac{\Delta t}{4} \ddot{\mathbf{u}}^{n}\right)\right]+\frac{\Delta t^{2}}{4}\left(\mathbf{f}^{n+1}+\mathbf{C} \theta^{n+1}\right)
$$

4. Solve: 


$$
\left(\mathbf{M}+\frac{\Delta t^{2}}{4} \mathbf{K}\right) \mathbf{u}^{n+1}=\mathbf{F}^{n+1^{*}}
$$

5. Update:

$$
\begin{aligned}
& \ddot{\mathbf{u}}^{n+1}=\mathbf{M}^{-1}\left(\mathbf{f}^{n+1}+\mathbf{C} \theta^{n+1}-\mathbf{K u}^{n+1}\right) \\
& \dot{\mathbf{u}}^{n+1}=\dot{\mathbf{u}}^{n}+\frac{\Delta t}{2}\left(\ddot{\mathbf{u}}^{n}+\ddot{\mathbf{u}}^{n+1}\right) \\
& \dot{\boldsymbol{\theta}}^{n+1}=\mathbf{Q}^{-1}\left(\mathbf{r}^{n+1}-\theta_{0} \mathbf{C}^{T} \dot{\mathbf{u}}^{n+1}-\mathbf{H} \theta^{n+1}\right)
\end{aligned}
$$

Equations (20) to (24) involve algebraic computations that are common to most implicit algorithms, when applied to the uncoupled problem. Only the quantity $\mathbf{C}^{T} \mathbf{M}^{-1} \mathbf{C}$ deserves special attention. In particular, it is important to note that:

- $\mathbf{C}^{T} \mathbf{M}^{-1} \mathbf{C}$ is not a full matrix. It is a symmetric banded operator. Let $n_{s}$, $n_{h}, b_{s}$ and $b_{h}$ denote the sizes and the semi-bandwidths of the structural and heat matrices, respectively. Typically, $n_{s}$ and $b_{s}$ are two to six times larger than $n_{h}$ and $b_{h}$. The matrix product $\mathbf{C}^{T} \mathbf{M}^{-1} \mathrm{C}$ is $n_{h}$ by $n_{h}$ and has a semibandwidth close to $2 b_{h}$. Therefore, equation (21) entails the solution of an $n_{h}$ by $n_{h}$ symmetric banded system. On the other hand, if equation (6) had been injected into equation (5) - that is, if the temperature field had been eliminated from the structural equation - the resulting augmentation term would have been $\mathrm{CQ}^{-1} \mathrm{C}^{T}$ which is $n_{s}$ by $n_{s}$ and has a semi-bandwidth close to $2 b s$. The latter would have entailed the solution of a symmetric system that is several times larger and denser than (21). For a rectilinear mesh composed of two-dimensional truss elements, the patterns of matrices $\mathbf{C}, \mathbf{C}^{T}, \mathbf{C}^{T} \mathbf{M}^{-1} \mathbf{C}$ and $\mathbf{C Q} \mathbf{Q}^{-1} \mathbf{C}^{T}$ are depicted in figure 1.

- the additional cost incurred by the augmentation term is restricted to the factorization and subsequent solutions of equation (21). The precise value of this additional cost (with respect to the conventional procedure (3)) depends on the cleverness of the implementation. 

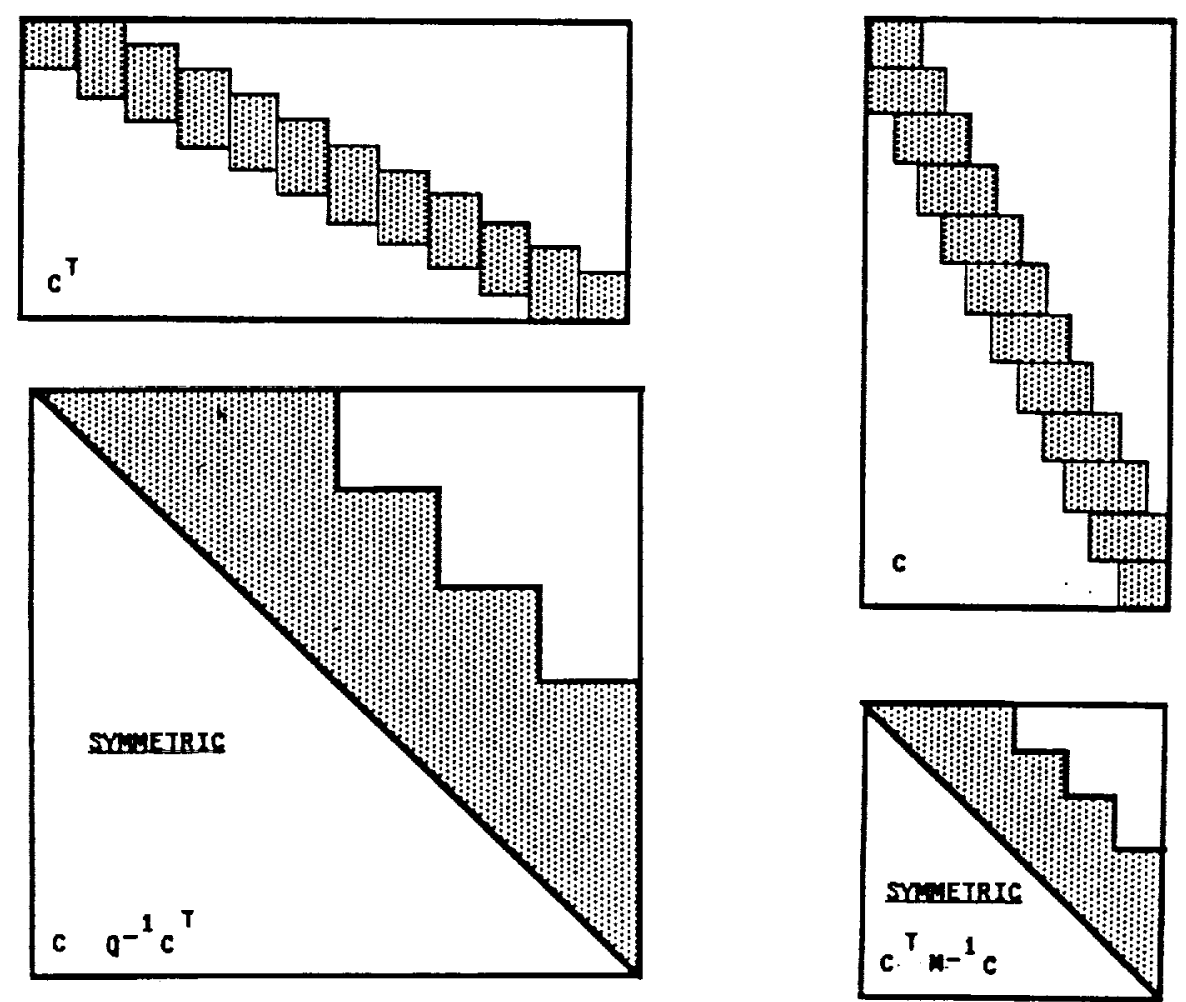

FIG. 1 Patterns of the coupling matrices for a rectilinear mesh with $2 D$ truss elements 
In order to illustrate the computational costs of the proposed numerical procedure, we consider the problem of a clamped square plate where the edges are exposed to a sudden heating. The finite element mesh is composed of $N$ by $N$ 4-node regular elements. The stiffness and conductivity matrices $\mathbf{K}$ and $\mathbf{Q}$ are assumed to be stored in banded form so that operation counting is facilitated. In practice, these matrices are compacted in skyline data structures. We denote by $d$ and $p$, respectively the number of structural degrees of freedom per node $(d \leq 6)$ and the number of integration steps.

The assumption of an $N$ by $N$ regular mesh with a number of fixed degrees of freedom at each node is unlikely in practice. However, it is the worst case as far as the computational effort required for the evaluation of the product $\mathbf{C}^{T} \mathbf{M}^{-1} \mathbf{C}$.

For the above problem, the formation and factorization of equations (21) and (23) require respectively $(2+d) N^{4}$ and $d^{3} N^{4} / 2$ multiplications. The resolution of equations (20)-(24) requires $\left(7 d^{2}+6 d+3\right) N^{3}$ multiplications for each time step. Therefore, the total computational effort needed for the transient coupled solution using the proposed stabilized procedure is:

$$
E^{s} \sim\left(\frac{d^{3}}{2}+d+2\right) N^{4}+p^{s}\left(7 d^{2}+6 d+3\right) N^{3}
$$

For the same problem, the computational cost associated with a conventional second-order accurate conditionally stable procedure (3) is:

$$
E^{c} \sim\left(\frac{d^{3}+1}{2}\right) N^{4}+p^{c}\left(7 d^{2}+6 d+3\right) N^{3}
$$

Clearly, unconditional stability is obtained at the cost of $(d+3 / 2) N^{4}$ additional floating point operations. For linear problems, this computational effort is needed once. In the following, we show that this overhead is compensated by a much larger time step.

The natural frequencies of the clamped square plate are given by:

$$
\omega_{m n}=\pi^{2} \sqrt{\frac{E l^{3}}{12\left(1-\nu^{2}\right) \rho}}\left(\frac{m^{2}+n^{2}}{a^{2}}\right)
$$

where $E, \nu, l$, and $a$ are respectively Young modulus, Poisson's coefficient, the plate thickness and its edge size [20]. Therefore, the lowest frequency is: 


$$
\omega_{\min }=\frac{2 \pi^{2}}{a^{2}} \sqrt{\frac{E l^{3}}{12\left(1-\nu^{2}\right) \rho}}
$$

and a good approximation of the highest element frequency is: .

$$
\omega_{\max }^{(e)}=\frac{2 \pi^{2}}{a^{2}} N^{2} \sqrt{\frac{E l^{3}}{12\left(1-\nu^{2}\right) \rho}}
$$

An adequate time step for the stabilized procedure is given by $\omega_{\min } \Delta t^{s}=\pi / 10$. For the conventional conditionally stable staggered procedure where both $\mathbf{u}$ and $\theta$ are integrated with the trapezoidal rule, the stable time step is expressed as a multiple of the time step based on the Courant condition associated with the hyperbolic structural equation. Hence, $\Delta t^{c}=m \times 2 / \omega_{\max }^{(e)}$, where $m \geq 1$. Using (28) and (29) we have:

$$
\begin{aligned}
\Delta t^{s} & =\frac{a^{2}}{20 \pi} \sqrt{\frac{12\left(1-\nu^{2}\right) \rho}{E l^{3}}} \\
\Delta t^{c} & =\frac{m a^{2}}{\pi^{2} N^{2}} \sqrt{\frac{12\left(1-\nu^{2}\right) \rho}{E l^{3}}}
\end{aligned}
$$

so that

$$
\begin{aligned}
& p^{s}=40 \\
& p^{c}=\frac{2 \pi N^{2}}{m}
\end{aligned}
$$

are the number of steps which would cover twice the largest period of the problem. The computational costs for both procedures become:

$$
\begin{aligned}
E^{s} \sim\left(\frac{d^{3}}{2}+d+2\right) N^{4} \\
E^{c} \sim \frac{2 \pi}{m}\left(7 d^{2}+6 d+3\right) N^{5}
\end{aligned}
$$

which demonstrates the superiority of the proposed stabilized staggered procedure for $N$ sufficiently large $(N>m / 14)$. 


\section{NUMERICAL EXAMPLES}

First, we consider the Second Danilovshaya problem [13]. An elastic half-space $(x>0)$ with the surface plane $x=0$ assumed free of tractions for all time is exposed to a sudden high ambient temperature $\theta_{\infty}$. The continuum is assumed to be mechanically constrained and thermally insulated so that the displacement and temperature fields are given by:

$$
\begin{aligned}
u_{x} & =u_{x}(x, t) \\
u_{y} & =0 \\
u_{z} & =0 \\
\theta & =\theta(x, t)
\end{aligned}
$$

The boundary and initial conditions for this problem are:

$$
\begin{aligned}
\sigma_{x x}(0, t) & =0 \\
k \frac{\partial \theta}{\partial x}(0, t) & =h\left(\theta(0, t)-\theta_{\infty}\right) \\
\text { and } & \\
u_{x}(x, 0) & =0 \\
\dot{u}_{x}(x, 0) & =0 \\
\theta(x, 0) & =\theta_{0}
\end{aligned}
$$

where $h$ is the boundary-layer conductance. The following dimensionless variables are introduced:

$$
\begin{aligned}
& \bar{x}=\frac{a x}{\kappa} \\
& \bar{t}=\frac{a^{2} t}{\kappa} \\
& \bar{\sigma}=\frac{\sigma_{x x}}{\beta \theta_{0}} \\
& \bar{\theta}=\frac{\theta-\theta_{0}}{\theta_{0}} \\
& \bar{u}=\frac{a(\lambda+2 \mu) u_{x}}{\kappa \beta \theta_{0}}
\end{aligned}
$$


where

$$
\begin{aligned}
\kappa & =\frac{k}{\rho c} \\
a^{2} & =\frac{\lambda+2 \mu}{\rho} \\
\beta & =\frac{\alpha}{3 \lambda+2 \mu}
\end{aligned}
$$

The thermomechanical coupling parameter is defined by:

$$
\delta=\frac{\beta^{2} \theta_{0}}{\rho c(\lambda+2 \mu)}=\frac{\beta^{2} \theta_{0}}{\rho^{2} a^{2} c}
$$

The exact solution for this problem can be obtained using the Laplace transform (see Nickell and Sackman [21]). The finite element solution is carried out using 2 -node linear elements. The ratio $\kappa h / a k$ is fixed to 0.5 and the thermomechanical coupling parameter $\delta$ is set to 1 . We report on the generated results for two time integration steps, $\Delta t^{(1)}=\pi / 5 \omega_{\min }$ and $\Delta t^{(2)}=\Delta t^{(1)} / 2=\pi / 10 \omega_{\min }$. These correspond to sampling the largest period of the mechanical problem into 10 and 20 steps, respectively. Figure 2 depicts the dimensionless temperature $\bar{\theta}$ at $\bar{x}=1.0$ as a function of the dimensionless time $\bar{t}$, for $\Delta t=\Delta t^{(1)}$. Figure 3 reports the dimensionless displacement $\bar{u}(\bar{t})$ at $\bar{x}=1.0$, for $\Delta t=\Delta t^{(2)}$. As expected, the results for $\Delta t=\Delta t^{(2)}$ are more accurate than those for $\Delta t=\Delta t^{(1)}$. However in both cases, the generated solutions are in good agreement with the exact ones. 


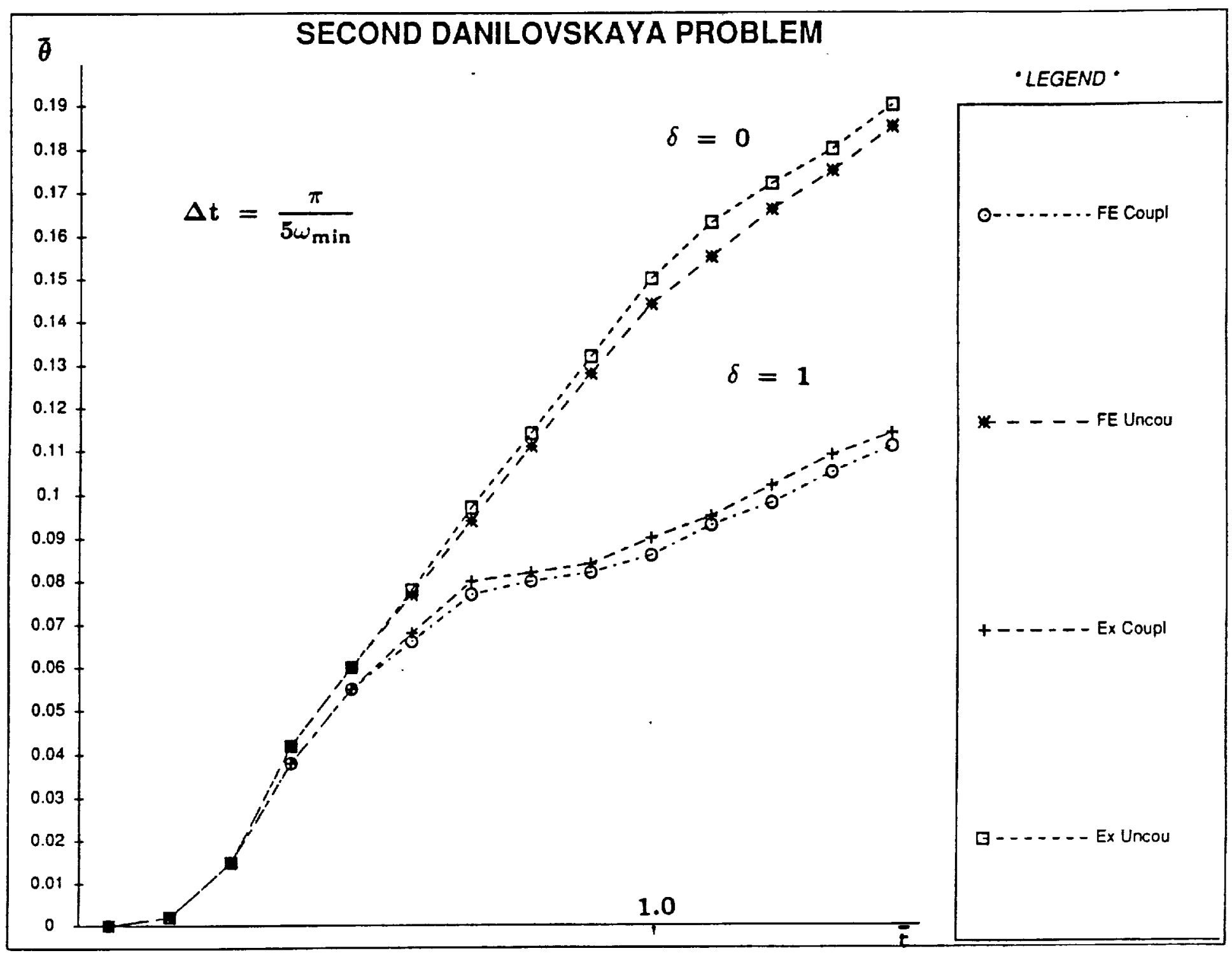

FIG. 2 Dimensionless temperature at $\bar{x}=1.0$ 


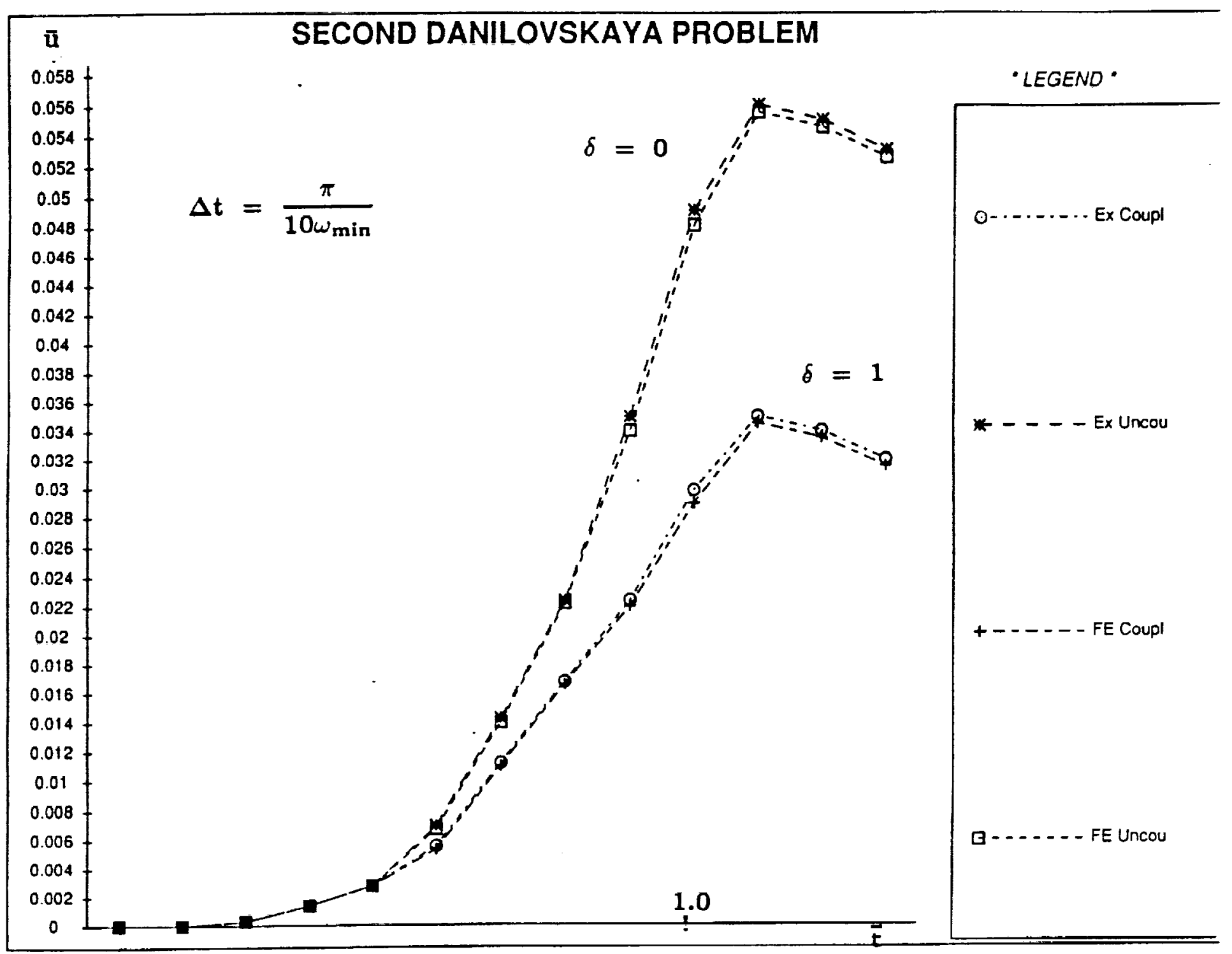

FIG. 3 Dimensionless displacement at $\bar{x}=1.0$ 
Next, we consider the case of an infinitely long elastic circular shaft of radius $R$, where the surface temperature undergoes a sudden uniform change over a finite band of length $Z$, and is steadily maintained thereafter (fig. 4). Youngdahl and Sternberg have presented in [14] an exact solution for the transient temperature and thermal stresses distributions in the shaft, when thermomechanical coupling is neglected, in the form of definite integrals and infinite series. In cylindrical co-ordinates $(r, \phi, z)$, the axisymmetric torsionless displacement and temperature fields are given as:

$$
\begin{aligned}
u_{r} & =u_{r}(r, z, t) \\
u_{\phi} & =0 \\
u_{z} & =u_{z}(r, z, t) \\
\theta & =\theta(r, z, t)
\end{aligned}
$$

The boundary and initial conditions for this problem are:

$$
\begin{aligned}
\sigma_{r r}(R, z, t) & =0 \\
\sigma_{r z}(R, z, t) & =0 \\
\sigma_{r r} & \rightarrow 0 \text { as }|z| \rightarrow \infty \\
\sigma_{\phi \phi} & \rightarrow 0 \text { as }|z| \rightarrow \infty \\
\sigma_{z z} & \rightarrow 0 \text { as }|z| \rightarrow \infty \\
\sigma_{r z} & \rightarrow 0 \text { as }|z| \rightarrow \infty \\
\theta(R, z, t) & =\theta_{\infty} \quad|z|<\frac{Z}{2} \\
\theta(R, z, t) & =0 \quad|z|>\frac{Z}{2}
\end{aligned}
$$

and

$$
\begin{gathered}
u_{r}(r, z, 0)=0 \\
u_{z}(r, z, 0)=0 \\
\dot{u}_{r}(r, z, 0)=0 \\
\dot{u}_{z}(r, z, 0)=0 \\
\theta(r, z, 0)=0
\end{gathered}
$$

The following new dimensionless variables (there should be no confusion over the 
present definition of these variables and their earlier use) are introduced:

$$
\begin{aligned}
& \rho=\frac{r}{R} \\
& \xi=\frac{2 z}{L} \\
& \bar{\theta}=\frac{\theta}{\theta_{\infty}} \\
& \bar{t}=\frac{k t}{R^{2}}
\end{aligned}
$$

For all computations, we set $L=2 R$ and $\nu=1 / 4$. The finite element solution is carried out using 4-node axisymmetric linear elements, and a time step $\Delta t=\pi / 10 w_{\min }$. Figure 5 compares the predicted temperatures at the center of the shaft $(\rho=0)$ with the exact ones for $\delta=0$, and reports on the effect of thermocoupling $(\delta=0.5)$ on temperature distribution. Clearly, the stabilized procedure provides accurate solutions. The variations of the radial stress at $\xi=0.1$ for $\delta=0$ and $\delta=0.5$ are depicted in figure 6 . All numerical results are reported at $\bar{t}=0.2$. It is interesting to note that when the thermocoupling effect is neglected the temperature field is overestimated, but the radial stress distribution is underestimated.
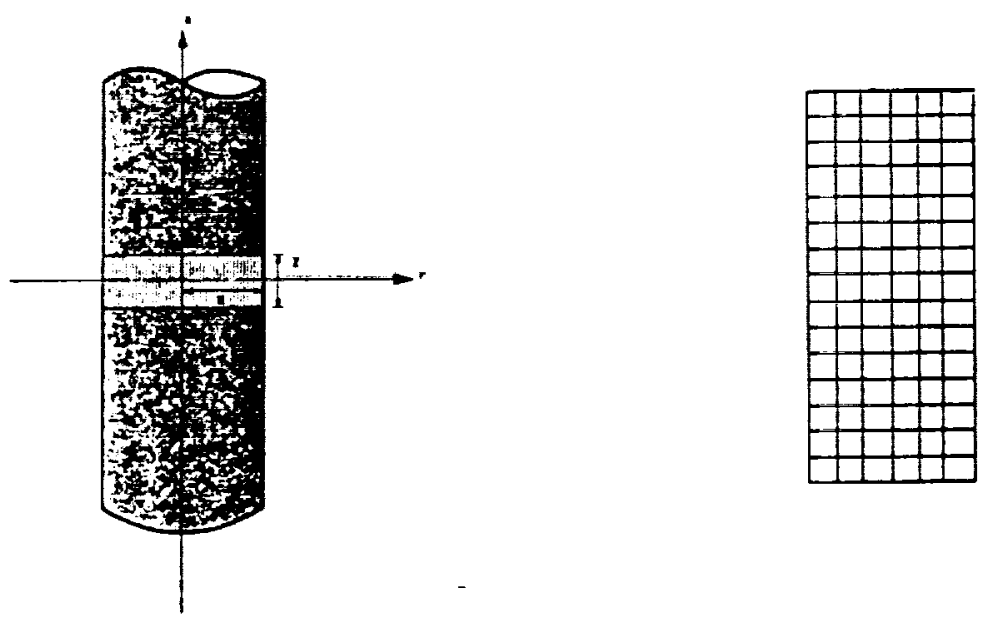

FIG. 4 Problem geometry and finite element discretization 


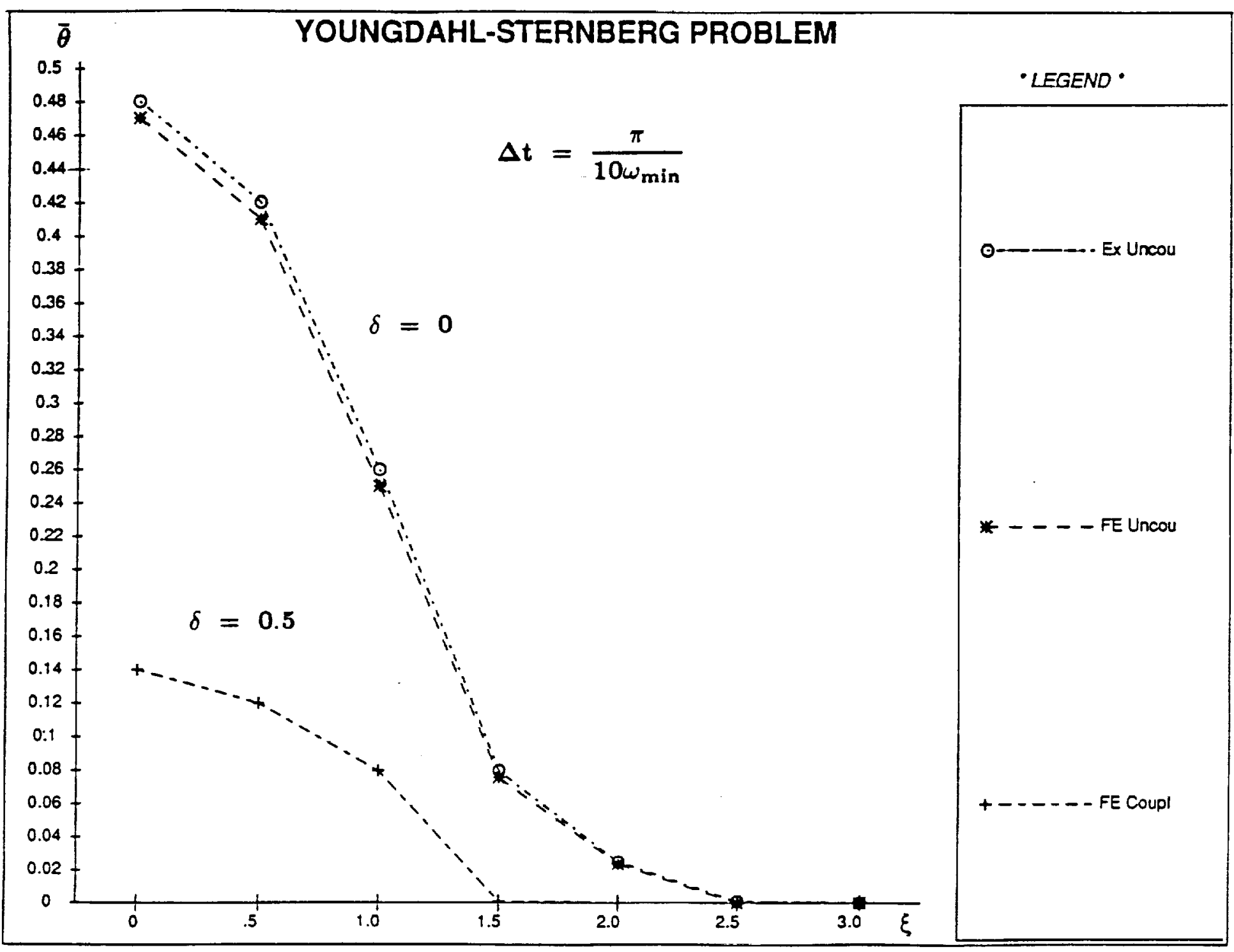

FIG. 5 Dimensionless temperat ure at $\rho=0$ for $\bar{t}=0.2$ 


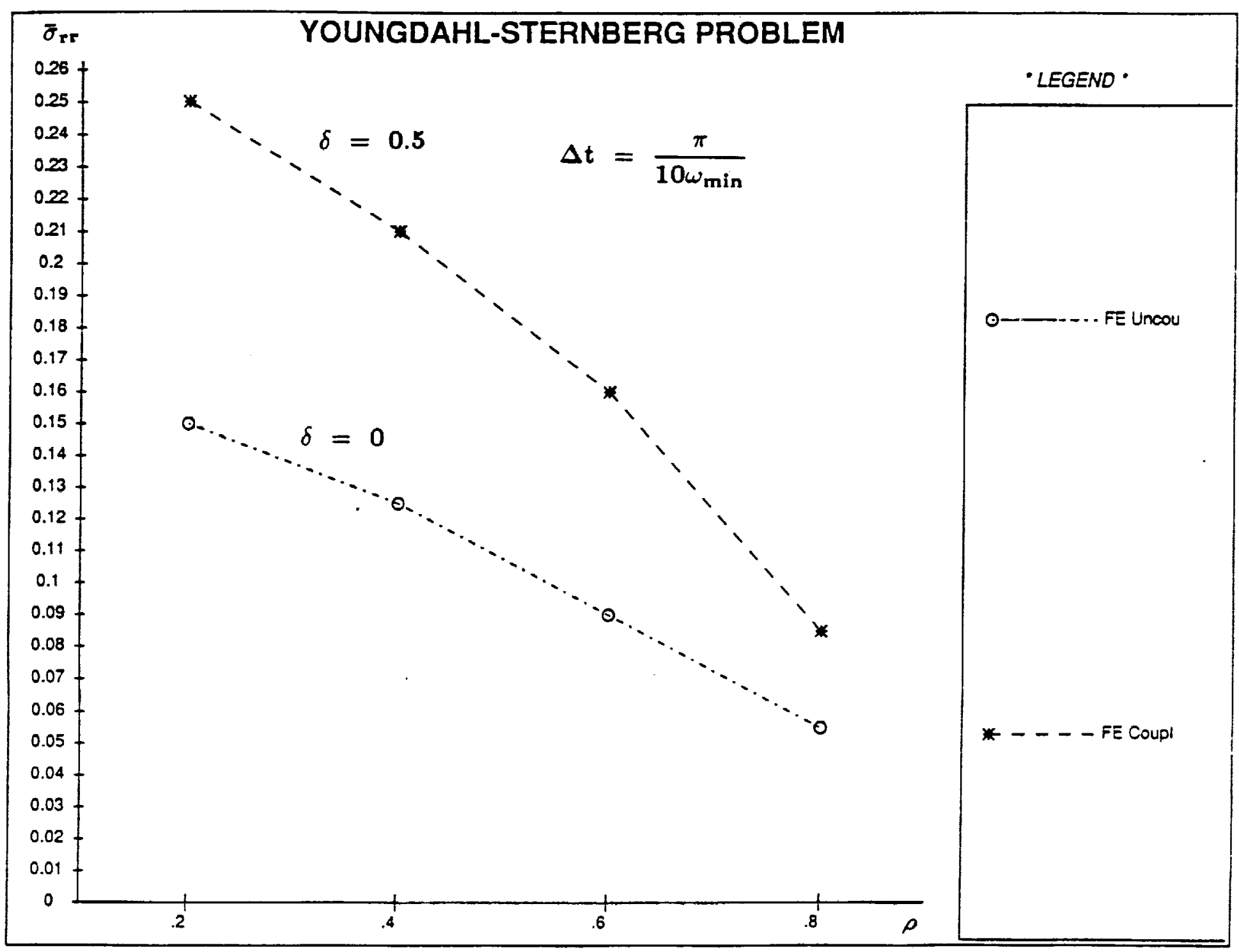

FIG. 6 Dimensionless radial stress at $\xi=0.1$ for $\bar{t}=0.2$ 


\section{CONCLUSION}

An implicit-implicit staggered procedure for the solution of thermoelastic problems is presented. It is stabilized with a cost-effective semi-algebraic augmentation scheme. The resulting transient algorithm is unconditionally stable and second-order accurate.

\section{ACKNOWLEDGMENT}

The authors would like to thank Professor Carlos Felippa at the University of Colorado, Boulder, for his guidance and interest in this work. The first author wishes to acknowledge the partial support of NASA Headquarters under grant number NAGW-1388. The second author acknowledges the support by NASA Lewis Research Center under grant NAG3-934.

\section{REFERENCES}

[1] E. L. Wilson and R. E. Nickell, "Application of the Finite Element Method to Heat Conduction Analysis", Nucl. Eng. Des., Vol. 4, pp. 276-286 (1966)

[2] R. E. Nickell and J. L. Sackman, "Approximate Solutions in Linear Coupled Thermoelasticity", J. Appl. Mech., Vol. 35, pp. 255-266 (1968)

[3] J. T. Oden, "Finite Element Analysis of Nonlinear Problems in the Dynamical Theory of Coupled Thermoelasticity", Nucl. Eng. Des., Vol. 10, pp. 465-475 (1969)

[4] J. T. Oden and W. H. Armstrong, "Analysis of Nonlinear Dynamic Coupled Thermoviscoelasticity Problems by the Finite Element Method", Computers \& Structures, Vol. 1, pp. 603-621 (1971)

[5] E. C. Ting and H. C. Chen, "A Unified Numerical Approach for Thermal Stress Waves", Computers \& Structures, Vol. 15, No. 2, pp. 165-175 (1982)

[6] W. K. Liu and Y. F. Zhang, "Unconditionally Stable Implicit-Explicit Algorithms for Coupled Thermal Stress Waves", Computers \& Structures, Vol. 17. No. 3, pp. 371-374 (1983)

[7] A. Wamberg, "Rational Runge Kutta Methods for Solving Systems of O. D. E.", Computing, Vol. 20, pp. 333-342 (1978)

[8] E. Hairen, "Unconditionally Stable Explicit Methods for Parabolic Equation", Numerical Math., Vol. 35, pp. 57-68 (1980) 
[9] W. K. Liu and H. G. Chang, "A Note on Numerical Analysis of Dynamic Coupled Thermoelasticity", J. Appl. Mech., Vol. 52, pp. 483-485 (1985)

[10] T. J. R. Hughes and T. Belytschko, "A Precis of Developments in Computational Methods for Transient Analysis", J. Appl. Mech., Vol. 50, pp. 1033-1041 (1983)

[11] K. C. Park, "Stabilization of Partitioned Solution Procedure for Pore FluidSoil Interaction Analysis", Int. J. Num. Meth. Eng., Vol. 19, pp. 1669-1673 (1983)

[12] O. C. Zienkiewicz, D. K. Paul and A. H. C. Chan, "Unconditionally Stable Staggered Solution Procedure for Soil-Pore Fluid Interaction Problems", Int. J. Num. Meth. Eng., Vol. 26, pp. 1039-1055 (1988)

[13] V. I. Danilovskaya, "On a Dynamical Problem of Thermoelasticity", Prikladnaya Matematika i Mechanika, Vol. 14, No. 3, pp. 341-344 (1952)

[14] C. K. Youngdahl and E. Sternberg, "Transient Thermal Stresses in a Circular Cylinder", J. Appl. Mech., Vol. 28, pp. 25-34 (1961)

[15] Y. Dubois-Pelerin, "Linear Thermomechanical Coupling by the Staggered Method", IREM Internal Report, Ecole Polytechnique Federale de Lausanne (1989)

[16] K. C. Park, C. A. Felippa and J. A. DeRuntz, "Stabilization of Staggered Solution Procedures for Fluid-Structure Interaction Analysis", Computational Methods for Fluid-Structure Interaction Problems, ed. by Belytshcko and Geers, ASME Applied Mechanics Symposia Series, AMD, Vol. 26, pp. 94-124 (1977)

[17] K. C. Park, "Partitioned Transient Analysis Procedures for Coupled-Field Problems: Stability Analysis", J. Appl. Mech., Vol. 47, No. 2, pp. 370-376 (1980)

[18] F. R. Gantmacher, "The Theory of Matrices", 2, Chelsea Publication Co., New York, pp. 190-196 (1959)

[19] R. Bellman, "Introduction to Matrix Analysis", 2nd ed., McGraw-Hill, New York, pp. 249-262 (1970)

[20] L. Meirovitch, "Analytical Methods in Vibrations", The Macmillan Company, New York, p. 185 (1967)

[21] R. E. Nickell and J. L. Sackman, "The Extended Ritz Method Applied to Transient Coupled Thermoelastic Boundary-Value Problems", Rep. No. 67-3, Structures and Materials Research, University of California at Berkeley (1967) Linear Coupled Thermoelasticity", J. Appl. Mech., Vol. 35, pp. 255-266 (1968) 


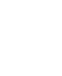


Public reporting burden for this collection of information is estimated to average 1 hour per response, including the time lor reviewing instructions. searching existing data sources, gathering and maintaining the data needed, and completing and reviewing the collection of information. Send comments regarding this burden estimate or any other aspect of this collection of information, including sugoestions for reducing this burden, to Washington Headquarters Services, Directorate for information Operations and Repons. 1215 Jellerson Davis Highway, Suite 1204, Arlington, VA 22202-4302, and to the Office of Management and Budget, Paperwork Peduction Project (0704-0188), Washington, DC 20503.

\begin{tabular}{|l|l|l|}
\hline 1. AGENCY USE ONLY (Leave blank) & $\begin{array}{r}\text { 2. REPORT DATE } \\
\text { November 1991 }\end{array}$ & $\begin{array}{r}\text { 3. REPORT TYPE AND DATES COVERED } \\
\text { Final Contractor Report-July } 89\end{array}$ \\
\hline
\end{tabular}

\section{TILE AND SUBTITE}

An Unconditionally Stable Staggered Algorithm for Transient Finite Element Analysis of Coupled Thermoelastic Problems

\section{AUTHOR(S)}

C. Farhat, K.C. Park, and Y. Dubois-Pelerin
5. FUNDING NUMBERS

WU-505-63-5B

G-NAGW-1388 and

NAG3-934

3. PERFORMING ORGANIZATION REPORT NUMBER

None

University of Colorado

Department of Aerospace Engineering Sciences and

Center for Space Structures and Controls

Boulder, Colorado 80309

9. SPONSORING/MONITORING AGENCY NAMES(S) AND ADDRESS(ES)

10. SPONSORING/MONTTORING

AGENCY REPORT NUMBER

National Aeronautics and Space Administration

Lewis Research Center

Cleveland, Ohio 44135 - 3191

NASA CR - 189066

\section{SUPPLEMENTARY NOTES}

Project Manager, C.C. Chamis, Structures Division, NASA Lewis Research Center, (216) 433-3252. C. Farhat and

K.C. Park, University of Colorado; Y. Dubois-Pelerin, Ecole Polytechnique Federale de Lausanne, Lausanne,

Switzerland.

\begin{tabular}{l|l} 
12a. DISTRIBUTION/AVAILABILTYY STATEMENT & 12b. DISTRIBUTION CODE
\end{tabular}

Unclassified - Unlimited

Subject Category 39

13. ABSTRACT (Maximum 200 mords)

An unconditionally stable second order accurate implicit-implicit staggered procedure for the finite element solution of fully coupled thermoelasticity transient problems is proposed. The procedure is stabilized with a semi-algebraic augmentation technique. A comparative cost analysis reveals the superiority of the proposed computational strategy to other conventional staggered procedures. Numerical examples of one and two-dimensional thermomechanical coupled problems demonstrate the accuracy of the proposed numerical solution algorithm.

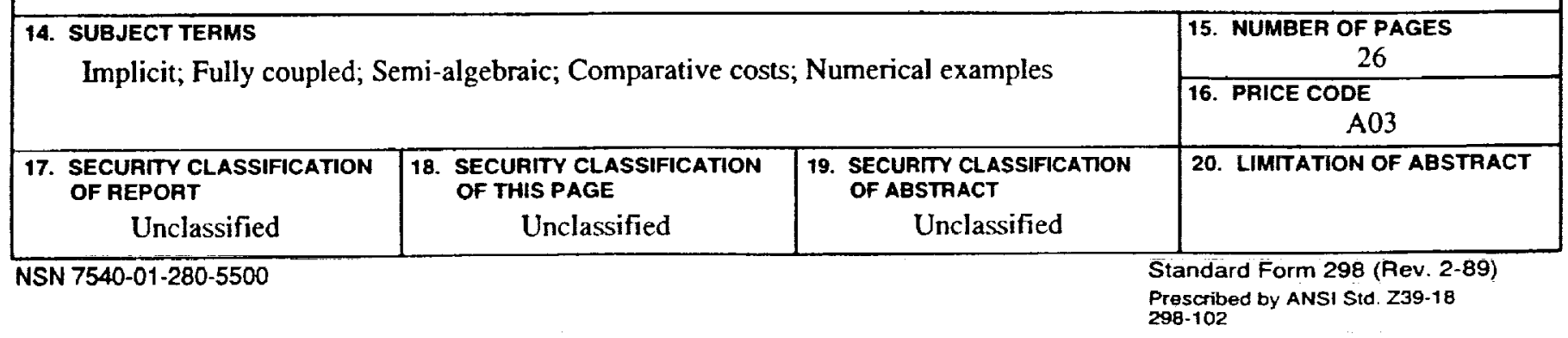

